\title{
Safety and efficacy of hyperbaric oxygen therapy in chronic wound management: current evidence
}

This article was published in the following Dove Press journal:

Chronic Wound Care Management and Research

22 April 2015

Number of times this article has been viewed

\section{Paul Eggleton' \\ Alexandra J Bishop ${ }^{2}$ \\ Gary R Smerdon²}

'Institute of Biomedical \& Clinical Science, University of Exeter Medical School, Exeter, ${ }^{2}$ DDRC Healthcare, Plymouth, UK
Correspondence: Paul Eggleton Institute of Biomedical \& Clinical Science, University of Exeter Medical School, St Luke's Campus, Heavitree Road, Exeter, Devon EXI 2LU, UK

Tel +44 I392 722940

Email p.eggleton@exeter.ac.uk

\begin{abstract}
The breathing of pure oxygen under pressure to treat tissue damage has been employed for almost 45 years and has been investigated in prospective, retrospective, and randomized controlled trials. The physiological effects of oxygen treatment on wound tissue are profound, and include activation of immune cells, changes in cytokine production, and modulation of inflammatory and bactericidal mediators. Hyperbaric oxygen influences the biochemistry of whole cells, altering cell proliferation, angiogenesis, clotting, and tissue regeneration. The precise effects of hyperbaric oxygen on individual cell types and tissues are only beginning to be revealed in both animal and human studies. Many independent studies using hyperbaric oxygen adjunctively with standard wound care have observed improved healing, in particular for diabetic foot ulcers, and can result in a significant reduction in major amputations. Side effects occur infrequently, but myopia, ear barotrauma, and rarely oxygen toxicity have been reported. As antibiotics become less available, and clinician time and complex dressings become more expensive, use of hyperbaric oxygen as a means of treating a variety of wound types may become an increasingly appropriate option for treatment.
\end{abstract}

Keywords: clinical trials, foot ulceration, non-healing wounds, oxygen

\section{Introduction}

Chronic wound ulcers can be categorized into four main groups, ie, venous, arterial, diabetic, and pressure/decubitus ulcers. All cause a significant financial and social burden worldwide. ${ }^{1,2}$ In 2009, approximately 6.5 million people required medical intervention for chronic wounds in the USA alone at a cost of US\$25 billion. ${ }^{3}$ The prevalence of so many chronic wounds has led to the view that there is a lack of consistency in chronic wound care practice. ${ }^{4}$ Chronic wounds can be difficult to heal, with patients often following a path that interchanges between improvement and deterioration without healing for many years, despite the use of conventional treatments. Hyperbaric oxygen therapy (HBOT) has been used as an accompaniment cure for chronic wounds for many years, although treatment is not easily accessible to many and often depends on geographic location, clinicians' understanding of the treatment, and evidence supporting its use.

\section{Pathophysiology of chronic non-healing wounds}

Most wounds go through a normal healing process consisting of three stages, ie, an acute inflammatory phase, a proliferative phase, and a remodeling phase. To improve wound care practice further, physiological studies are required to assess the various treatment practices employed. This is also the case for use of HBOT. Chronic wounds 
are susceptible to infection, resulting in a prolonged inflammatory phase linked to tissue damage provoked by increased free radical production and proteolytic activity. These inflammatory mediators can lead to an imbalance in growth factors, cytokines, and chemokines released into the tissue, triggering ischemia, edema, and inhibition of entry of nutrients into the wound.

\section{Role of oxygen in wound healing}

Oxygen is important during normal wound healing, with hypoxic conditions triggering healing processes, and large amounts of oxygen being consumed. The initial inflammatory phase of wound healing involves hemostasis to prevent excessive blood loss. This is instigated by activation of the clotting cascade by injured cells, and involves cleavage of fibrinogen to fibrin by thrombin. The fibrin forms a stable clot and then the basic wound matrix, and traps activated platelets that release the contents of $\alpha$-granules, including the various cytokines and growth factors responsible for induction of the inflammatory phase. ${ }^{5}$ We have investigated the role of hyperbaric oxygen in human platelet activation. When platelets were exposed to hyperbaric oxygen $(97.7 \%$ $\mathrm{O}_{2}$, balance $\mathrm{CO}_{2}$ at 2.2 absolute atmospheres [ATA]) we observed a six fold increase in platelet aggregation and more protein release, including chaperone 14-3-3-zeta and $\alpha$-2-macroglobulin, both of which are involved in apoptosis. ${ }^{6}$ Within 6 hours of injury, numerous inflammatory growth factors are released, which aid the recruitment of neutrophils and subsequently macrophages to the wound site. Many of these processes require extra cell metabolism for biosynthesis and transport of proteins, which requires oxygen.

During the proliferative phase, approximately 2 days into wound healing, macrophages continue to express growth factors, many of which stimulate angiogenesis and formation of granulation tissue comprising of endothelial cells, fibroblasts, and inflammatory cells. Then, during the migration and angiogenic step of the proliferation phase, these cells migrate into the wound and continue to repair tissue. High levels of oxygen are required for this proliferation phase, especially when reepithelialization occurs surrounding the wound in conjunction with keratinocytes to seal the wound from the extracellular environment. We have investigated the effects of hyperbaric oxygen on inflammatory cell (neutrophil)-endothelial cell interactions under hypoxic and chronic wound conditions, and found that hyperbaric oxygen reduces inflammatory cellendothelial cell interaction, possibly reducing local inflammation during revascularization of wound tissue. ${ }^{7}$ The hypoxic conditions in the damaged vascularized wound area trigger in part the production of transcription factors such as hypoxiainducible factor 1- $\alpha$ and vascular endothelial growth factor by endothelial cells and macrophages. As the macrophages remove necrotic cellular debris, the phagocytic processes trigger production of reactive oxygen species, which requires significant amounts of oxygen. Finally, during the remodeling phase, oxygen is essential to aid in the removal of granulation tissue via the process of apoptosis. Oxygen is also required for the synthesis of type I collagen and its organization into bundles, replacing the type III collagen that was originally laid down in the granulation tissue, which ultimately enhances wound tensile strength.

Thus oxygen plays an important role in all cellular processes during wound healing, including cell metabolism, proliferation, and revascularization. Oxygen is also essential for increased antimicrobial activity, growth factor signal transduction, and collagen synthesis. It is therefore of great interest to know how infusing wound tissues with oxygen under pressure might be beneficial to the healing process. The partial pressure of oxygen $\left(\mathrm{pO}_{2}\right)$ in normal subcutaneous tissue is in the range of $40-80 \mathrm{mmHg}$, but drops to $<20 \mathrm{mmHg}$ under chronic wound conditions (Figure 1). Despite the growing body of evidence indicating the usefulness of HBOT in wound healing, the mechanistic regulation of wound physiology by HBOT is still in its infancy.

\section{Benefits of HBOT in chronic wounds}

Various international HBOT associations, for example the Undersea and Hyperbaric Medical Society and the European Committee for Hyperbaric Medicine, recommend the use of HBOT for a number of indications, including various

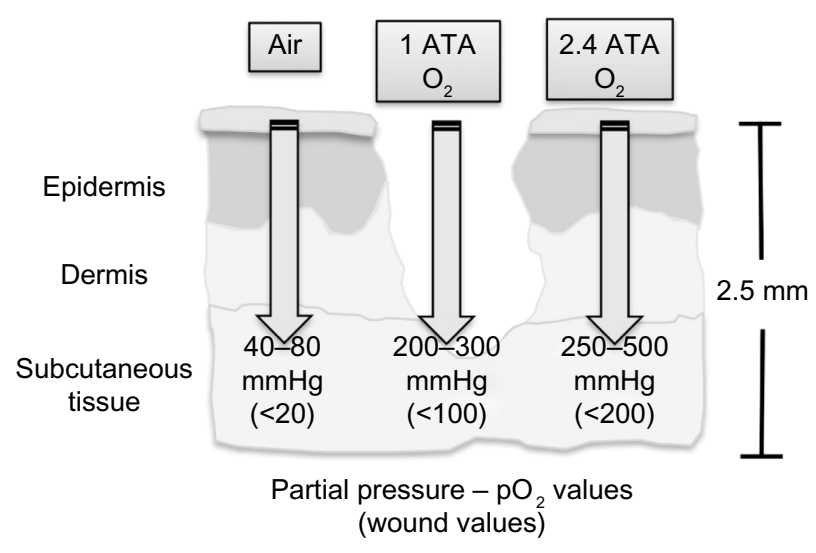

Figure I Partial pressure of oxygen in normal and wounded subcutaneous tissue upon exposure to air, oxygen, and hyperbaric oxygen.

Abbreviations: ATA, absolute atmospheres; $\mathrm{pO}_{2}$, partial pressure of oxygen. 
Table I Approved clinical indications for hyperbaric oxygen therapy

\begin{tabular}{|c|c|}
\hline Clinical condition & Definition \\
\hline Gas embolism & Entry of gas bubbles into arteries or veins \\
\hline Carbon monoxide & Hypoxic stress by elevated \\
\hline poisoning & carboxyhemoglobin \\
\hline Gas gangrene & Toxemia, edema, and necrotic tissue \\
\hline Crush injury & Trauma to multiple tissues \\
\hline Decompression sickness & $\begin{array}{l}\text { Supersaturation of tissue with inert gas } \\
\text { causing organ damage }\end{array}$ \\
\hline Arterial inefficiencies & $\begin{array}{l}\text { Hypoxia, persistent infection, and cellular } \\
\text { failure }\end{array}$ \\
\hline Severe anemia & Loss of red blood cell mass \\
\hline Intracranial abscess & $\begin{array}{l}\text { Brain abscess normally deep-seated, with } \\
\text { infection and immune cell involvement }\end{array}$ \\
\hline $\begin{array}{l}\text { Necrotizing soft tissue } \\
\text { infection }\end{array}$ & $\begin{array}{l}\text { Tissue necrosis due to infection, cellulitis, } \\
\text { and fasciitis with impaired immunity }\end{array}$ \\
\hline Osteomyelitis & Infection of bone \\
\hline Delayed radiation injury & Cellular toxicity caused by radiotherapy \\
\hline $\begin{array}{l}\text { Compromised grafts } \\
\text { and flaps }\end{array}$ & $\begin{array}{l}\text { Areas compromised by hypoxia or } \\
\text { decreased blood perfusion }\end{array}$ \\
\hline Acute thermal burns & $\begin{array}{l}\text { Compromised tissue unable to supply } \\
\text { oxygen and nutrients to surrounding tissue }\end{array}$ \\
\hline $\begin{array}{l}\text { Idiopathic sudden } \\
\text { hearing loss }\end{array}$ & $\begin{array}{l}\text { Loss of oxygen concentration in the } \\
\text { cochlea }\end{array}$ \\
\hline
\end{tabular}

types of chronic wounds and other clinical conditions (Table 1). During HBOT, patients are placed in a multiplace or monoplace chamber where they are required to breath $100 \%$ oxygen intermittently, at a pressure greater than sea level (ie, $>1$ ATA). The duration and frequency of treatment with HBOT and the ATA employed is often open for debate, but the pressurization used is normally 1.4 ATA or higher. The units used to measure $\mathrm{pO}_{2}$ in healthy and wound tissue are often quoted in $\mathrm{mmHg}$, kilopascals, or bar pressure (Table 2) before and after HBOT.

Patients with chronic wounds selected for HBOT usually have a history of nonresponsiveness to conventional treatments, including antibiotics and topical dressings, and failed debridement. Patient selection for HBOT can be assisted by noninvasive transcutaneous oxygen pressure $\left(\mathrm{TcpO}_{2}\right)$

Table 2 Units used to monitor partial pressure of oxygen and some commonly cited values for subcutaneous healthy and wound tissues

\begin{tabular}{llll}
\hline Condition & Bar $\left(\mathbf{k g f} / \mathbf{c m}^{2}\right)$ & $\mathbf{~ m m H g}$ (Torr) & $\mathbf{~ k P a}$ \\
\hline Normoxia & $\mathrm{I} .0$ & 760 & 101.32 \\
$\mathrm{PO}_{2}$ in chronic wounds & $0.066-0.026$ & $5-20$ & $0.66-2.66$ \\
$\mathrm{PO}_{2}$ in healthy tissue & 0.053 & $40-80$ & $5.33-10.66$ \\
$\mathrm{HBO}$ & 2.4 & 1,800 & 240 \\
$\mathrm{PO}_{2}$ in chronic wounds & $<0.27$ & $<200$ & $<26.66$ \\
$\mathrm{PO}_{2}$ in healthy tissue & $0.33-0.66$ & $250-500$ & $33.33-66.66$ \\
\hline
\end{tabular}

Abbreviations: $\mathrm{HBO}$, hyperbaric oxygen; $\mathrm{pO}_{2}$, partial pressure of oxygen. monitoring. This technique has recently been recommended for long-term monitoring of the efficacy of HBOT and in other clinical settings. ${ }^{8}$ One of the major functions of HBOT is to supply wound tissue with sufficient oxygen to encourage metabolism in the wound environment, especially when the vascular supply is impaired. Fife et al determined the predictive value of $\mathrm{TcpO}_{2}$ in envisaging the likelihood of benefit of HBOT in a large cohort $(n=1,114)$ of diabetic patients with a variety of lower extremity wounds ${ }^{9}$ graded on the Wagner scale. ${ }^{10}$ Based on this and other studies, current protocols recommend that patients with wound $\mathrm{pO}_{2}<40 \mathrm{mmHg}$ (hypoxic) while breathing air at 1 ATA that increases to about $100 \mathrm{mmHg}$ while breathing oxygen at 1 ATA are suitable candidates for HBOT. Whenever possible, the patients are tested at pressure inside a hyperbaric oxygen chamber, and if their wound $\mathrm{pO}_{2}$ values increase to $>200 \mathrm{mmHg}, 74 \%$ of patients are predicted to respond well to HBOT.

Patients with problem non-healing wounds attending the DDRC Healthcare facility (http://www.ddrc.org/) in Plymouth, UK, are treated using the protocol outlined in Royal Navy Treatment Table 66 (Figure 2). The patients enter the chamber and are pressurized during a 10-minute descent to 2.4 ATA. The patient remains at this pressure for 90 minutes, before being decompressed back to 1 ATA over a 10-minute period. Patients breathe oxygen during three 30-minute cycles, and to minimize the risk of oxygen toxicity, patients have 5-minute "air breaks" after 30 and 65 minutes, during which they remain under pressure but breathe air instead of $100 \%$ oxygen (Figure 2). The procedure itself is relatively safe, and our experience of treating patients over two decades has demonstrated the

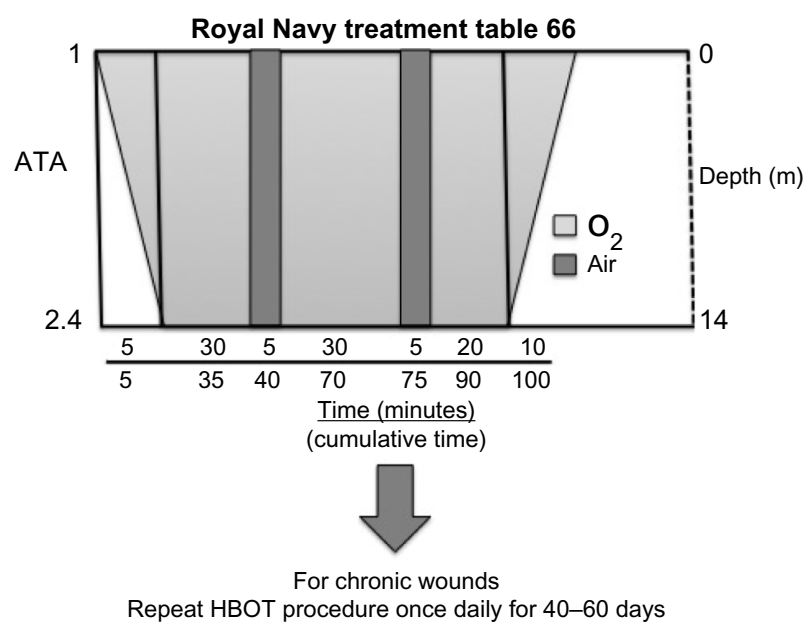

Figure 2 HBOT protocol for treatment of chronic wounds. Abbreviations: ATA, absolute atmospheres; HBOT, hyperbaric oxygen therapy. 
most common side effects to be mild barotrauma and myopia.

\section{Effects of hyperbaric oxygen on wound healing in humans}

The wound environment is a complex and dynamic one, and the overall objective of HBOT is to increase the diffusion gradient of oxygen in subcutaneous tissue by about 10-20fold to allow hyperoxygenation of ischemic tissue, reduce the levels of inflammatory cytokines, and stimulate the production of growth factors. In addition, HBOT enhances antibacterial activity, including production of oxygen free radicals, whilst reducing nonspecific activation of inflammatory cells. Further functions of HBOT are to promote transmigration of stem cells to infected wound tissue, alter leukocyte-endothelial cell adhesion, and promote collagen formation. There are numerous animal and clinical studies supporting these functions, and are described below.

Clinical evidence supports the concept that HBOT promotes the vascularization of wounds. A subset of bone marrow-derived stem/progenitor cells called endothelial progenitor cells have the ability to transmigrate to wounds, where they rapidly differentiate into mature endothelial cells and assist in vascular repair. In a study of eight diabetic patients treated with hyperbaric oxygen for a single 2-hour period at a pressure of 2.0 ATA on 6 consecutive days for up to 20 treatments, a significant but temporary increase in both stem/progenitor cells and endothelial progenitor cells was observed in the patients' blood after one, ten, and 20 treatments with HBOT. ${ }^{11}$ In the same study, biopsies of wound tissue revealed a marked elevation in the presence of $\mathrm{CD}_{133^{+}}$stem/progenitor cells, indicating that HBOT stimulates vasculogenic stem cell mobilization. With evidence of increased vascularization in wounds post-HBOT, the possibility of increased infiltration of proinflammatory cells such as neutrophils is of concern. However, it has been shown that HBOT inhibits adhesion of leukocytes to injured endothelium by inhibiting integrin expression. ${ }^{12}$ Our own studies have determined that changes in endothelial cell adhesion molecules are altered by a single HBOT treatment via nitric oxide-mediated mechanisms. ${ }^{7}$ In addition, we have shown that a single dose of HBOT enhances generation of reactive oxygen species and phagocytosis by neutrophils. Although this could lead to nonspecific inflammation at the site of a wound, it may also help prevent microbial colonization. HBOT also elicits enhanced apoptosis of phagocytes, thus offsetting the accumulation of potentially necrotic cell debris. ${ }^{13}$ Patients with type 2 diabetes are known to have impaired wound healing of ulcers, and HBOT consisting of three 25 -minute periods of $100 \%$ oxygen at a pressure of 2.4 ATA, interspersed with 5-minute periods of breathing air, for 6 days/week over 5-6 weeks has been shown to progressively reduce wound areas in diabetic patients $(n=18)$ by enhancing production and secretion of type I and III procollagens by fibroblast, which can be detected in plasma and help increase the tensile strength of wounds. ${ }^{14}$

From the evidence presented above, HBOT appears to be useful in promoting wound healing in a variety of ways that can affect the physiology of the wound even after the treatment has ceased. Limited HBOT research has been conducted using animal models, and has revealed a number of physiological pathways of wound healing affected by HBOT. However additional research is required, especially human trials or the use of human tissues, in order to advance our knowledge of the usefulness and safety of this treatment.

\section{Safety and efficacy of HBOT}

As presented in Table 1, the Undersea and Hyperbaric Medical Society currently identifies 14 disease states (indications) for HBOT, of which "enhancement of healing in selected problem wounds" falls within the clinical condition termed "arterial insufficiencies". Two types of chambers (monoplace and multiplace) are commonly used to administer HBOT to patients (Figure 3A and B). Monoplace chambers permit only single occupancy and in modern chambers the patient is moved in and out of the chamber on a sliding stretcher. The chamber walls are made of clear acrylic, providing high visibility and helping to reduce claustrophobia. Monoplace chambers are normally compressed with $100 \%$ oxygen, with the patients simply breathing the atmosphere within the chamber. Air breaks, if required, are administered by the patient holding an air-delivering built-in breathing system mask to the face. Multiplace chambers can accommodate multiple patients (ten patients are not uncommon), and patients can enter the chamber on foot if appropriate. The walls of the multiplace chamber are of steel construction. The chamber is compressed with air and the patient breathes $100 \%$ oxygen under pressure administered by means of a built-in breathing system mask or a hood. HBOT describes the systemic delivery of hyperbaric oxygen and should not be confused with normobaric (ambient pressure) oxygen delivery or topical oxygen therapy. The pressures used to deliver hyperbaric oxygen do not exceed 3 ATA (equivalent to a depth of $20 \mathrm{~m}$ in seawater) and the duration of treatment for elective therapies does not generally exceed 2 hours. Within these parameters, HBOT is considered a safe treatment 
A

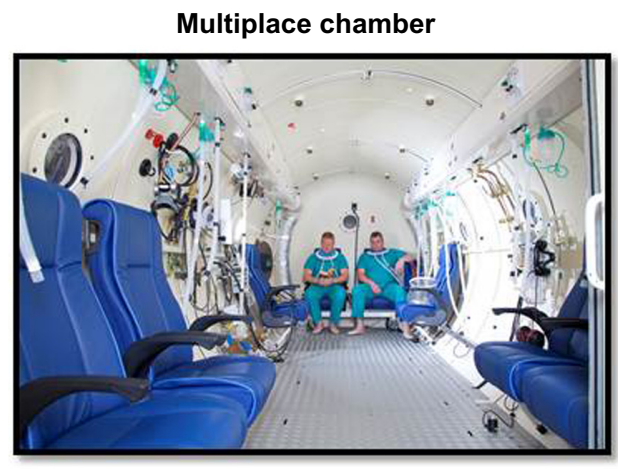

B

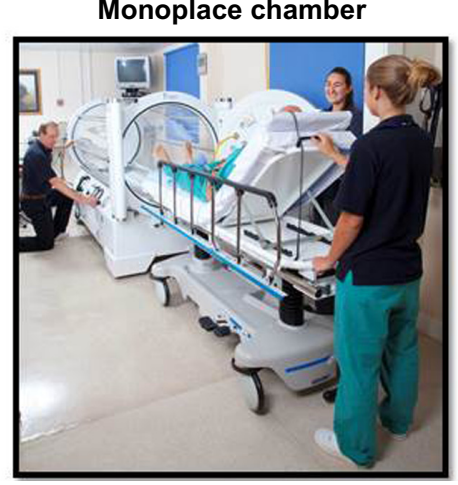

C

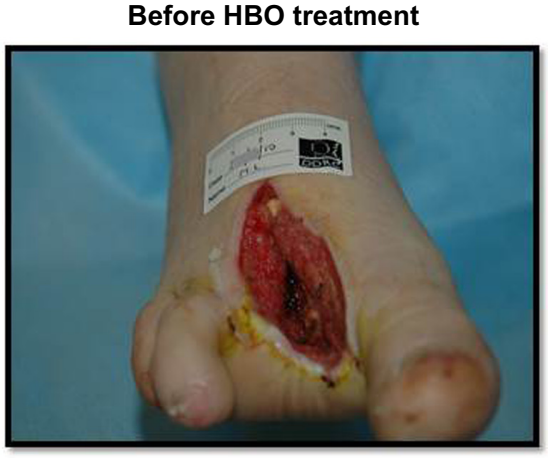

D

Post 34 HBO treatments

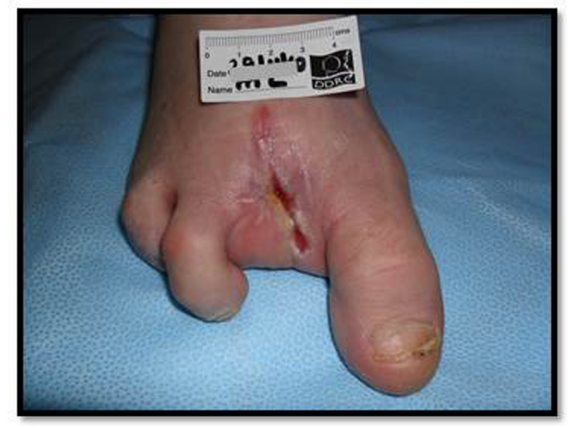

Figure 3 Patient HBO chambers and effect of $\mathrm{HBO}$ on components of wound healing. (A and B) Multiplace and monoplace chambers used to treat chronic wounds. (C) Typical type 2 diabetic foot sepsis leading to third digit amputation secondary to osteomyelitis. (D) Effect of a complete course of 36 sessions of HBO therapy over a 2-month period.

Abbreviation: $\mathrm{HBO}$, hyperbaric oxygen.

modality, but does carry some risks due to hyperoxia and increased pressure. Absolute contraindications to HBOT are limited to untreated pneumothorax and treatment with certain chemotherapeutic drugs (doxorubicin, cisplatin, and bleomycin). Relative contraindications include upper respiratory tract infections, chronic obstructive pulmonary disease, history of thoracic or ear surgery, hyperthermia, pregnancy, and claustrophobia.

The most common side effect of hyperoxia is a progressive myopia in patients undergoing prolonged periods of daily hyperbaric oxygen. ${ }^{15}$ The exact mechanism for this change in sight remains obscure but appears to be of lenticular origin ${ }^{16}$ and usually reverses a few days to several weeks after the last therapy. ${ }^{17}$ Central nervous system and pulmonary oxygen toxicity are rare, given that oxygen tolerance limits that avoid these manifestations are well defined. ${ }^{18-20}$ Middle ear barotrauma is the most common side effect of increased pressure. Incidence rates vary widely but can minimized by training in equalization techniques (Valsalva maneuver, yawning, jaw thrusting), vigilance on the part of the chamber attendant, or by tympanostomy tubes. ${ }^{21}$ Claustrophobia may cause some degree of anxiety within the confined spaces of a hyperbaric chamber, and occasionally mild sedatives are employed to allow some individuals to undertake or continue HBOT.

\section{Mechanisms of action in preclinical studies}

Whilst the use of oxygen at elevated pressures was first proposed for the treatment of decompression injury, ${ }^{22}$ its use for treatment of disease was only initiated when ChurchillDavidson et al described the use of elevated pressures and oxygen to potentiate radiotherapy in cancer patients in $1955 .{ }^{23}$ The following year, Boerema et al published a paper on the clinical use of hyperbaric oxygen to extend the duration of circulatory arrest during cardiac surgery. ${ }^{24}$ These publications were followed by reports of favorable clinical response to HBOT in patients suffering from clostridial infections ${ }^{25}$ and those poisoned by carbon monoxide. ${ }^{26}$ Within less than a decade, a diversity of medical disciplines were supporting the use of high oxygen tensions at pressure for the treatment of various disease states.

The development of HBOT initially progressed through clinical application and observation supported by some clinical trials, and as such there are limited animal studies determining efficacy. The majority of preclinical studies are 
laboratory-based and set out to determine the mechanisms of action of elevated oxygen levels rather than efficacy, and this is in contrast to the normal pathway of drug development in the modern era. Elective HBOT has arguably been most effectively applied to nonhealing wound repair in chronic diabetic ulcers (Figure 3C and D) and delayed post-radiation tissue injuries. These pathophysiologically distinct disorders share many common elements, including chronic inflammation, reduced oxygen supply, stromal cell depletion, and fibrosis. ${ }^{27-29}$ HBOT has been shown to promote angiogenesis, enhance fibroblast activity, augment formation of granulation tissue, reduce edema, and improve leukocyte function. ${ }^{30,31}$ Vasculogenesis is enhanced by HBOT-induced mobilization of stem cells from bone marrow. . $^{25,32,33}$

HBOT has been demonstrated to have bacteriostatic and bactericidal effects. With obligate anaerobes, the effect is rapid as a result of the sensitivity of these organisms to oxygen free radicals due to a lack of scavenging enzymes. Our work has demonstrated both pressure and hyperoxia to be important in the interaction between bacteria and neutrophillike cells. ${ }^{13}$ Antimicrobial activity is potentially detrimental to wound healing through development of a pro-inflammatory environment, but enhanced apoptosis can resolve the inflammation and support the progression of wound healing. ${ }^{13}$ In chronic wounds with an osteogenic component (including osteomyelitis), there is a growing body of evidence to support the promotion of bone repair with HBOT. Osteoblast stimulation, anti-osteoclastic effects, and bone regeneration have been demonstrated using intermittent oxygen supplementation, providing mechanistic evidence supporting the adjunctive use of HBOT. ${ }^{34}$

The use of HBOT for chronic wounds remains an inexact science. Research over the past 20 years has provided robust evidence describing the mechanisms of action and a greater understanding of the potential benefits. There remains a paucity of data relating to oxygen dose in both preclinical studies and clinical trials and an incomplete understanding of the relationship between elevated oxygen per se and the minimally toxic dose necessary to enhance healing mechanisms promoting wound closure and functional restoration of damaged tissues. Further research and an increased awareness of the relative safety of HBOT are required if the true potential of this sometimes controversial treatment modality is to be realized.

\section{Current clinical trial evidence for using HBOT to treat wounds}

Many studies of chronic wounds and HBOT have been undertaken over the past 45 years with respect to treating arterial, pressure/decubitus, venous, and diabetic ulcers. Kranke et al performed a Cochrane review of HBOT for chronic wounds, identifying 27 potentially eligible trials, but excluded 17 based on quality. ${ }^{35}$ They found no research on arterial or pressure ulcers that was eligible for inclusion in their systematic review, but found seven trials comparing HBOT as a treatment for diabetic ulcers with controls, ${ }^{36-42}$ one study comparing HBOT with extracorporeal shockwave therapy, ${ }^{43}$ and one study on HBOT for venous ulcers. ${ }^{44}$ Kranke et al concluded that there was some evidence that HBOT used adjunctively for diabetic wounds results in significant short-term improvement of wound healing by 6 weeks. ${ }^{35}$ The effect of HBOT on amputation rate could not be confirmed due to low participant numbers and poor reporting in studies. Cochrane reviews consider only randomized controlled trials. While these provide high-quality evidence, they can cause recruitment problems for researchers and are not always applicable to patients seen in clinics due to restrictive inclusion criteria.

The studies described here include prospective trials, irrespective of whether participants were randomized or whether the study had a control arm, and retrospective studies. The latter can be representative of what patients are experiencing and the outcomes seen in clinical practice without the restrictions required for prospective research. The wound types in each study are shown in Table 3 and demonstrate that the majority of published trials employing HBOT to treat chronic wounds have been performed in diabetic wounds (eleven prospective and eleven retrospective). One retrospective study assessed the outcome of both diabetic and venous ulcers, there was one prospective trial on venous ulcers alone, and then one prospective study on pressure ulcers. To our knowledge, no studies of hyperbaric oxygen for arterial ulcers have been performed, which is consistent with the findings reported above. ${ }^{35}$

\section{Pressure ulcers}

Rosenthal and Schurman used HBOT to treat pressure ulcers. ${ }^{45}$ Their study was reported by Hunter et al. ${ }^{46}$ They treated 18 participants (39 ulcers) with HBOT and had three control subjects (six ulcers). Randomization, blinding, number of treatments, and treatment pressure used are unclear. Complete healing was reported in 22 patients $(58 \%)$ and five $(13 \%)$ had a $>50 \%$ reduction in wound size. In comparison, none of the wounds in the control patients healed or reduced in size by $>50 \%$. While these results seem encouraging, the lack of information about the methodology used renders these findings unreliable. Adequate 
Table 3 Clinical studies using hyperbaric oxygen to treat chronic wounds

\begin{tabular}{|c|c|c|c|}
\hline Reference & Year & Study type & Wound type \\
\hline \multicolumn{4}{|l|}{ Prospective studies } \\
\hline Rosenthal and Schurman ${ }^{45}$ & |97| & CT & Pressure \\
\hline Baroni et $\mathrm{al}^{48}$ & 1987 & $\mathrm{CT}$ & Diabetic \\
\hline Oriani et al ${ }^{49}$ & 1990 & CT & Diabetic \\
\hline Doctor et $\mathrm{al}^{37}$ & 1992 & $\mathrm{RCT}$ & Diabetic \\
\hline Hammarlund and Sunberg ${ }^{44}$ & 1994 & $\mathrm{RCT}$ & Venous \\
\hline Faglia et $\mathrm{a}^{39}$ & 1996 & RCT & Diabetic \\
\hline Zamboni et $\mathrm{al}^{52}$ & 1997 & $\mathrm{CT}$ & Diabetic \\
\hline Kalani et $\mathrm{a}^{53}$ & 2002 & $\mathrm{CT}$ & Diabetic \\
\hline Abidia et $\mathrm{al}^{36}$ & 2003 & RCT & Diabetic \\
\hline Kessler et al ${ }^{40}$ & 2003 & $\mathrm{RCT}$ & Diabetic \\
\hline Duzgun et $\mathrm{a}^{38}$ & 2008 & RCT & Diabetic \\
\hline Löndahl et $\mathrm{al}^{41}$ & 2010 & $\mathrm{RCT}$ & Diabetic \\
\hline Wang et $\mathrm{al}^{43}$ & 2011 & $\mathrm{RT} / \mathrm{HBO} / \mathrm{ESWT}$ & Diabetic \\
\hline \multicolumn{4}{|l|}{ Retrospective studies } \\
\hline Ma et $\mathrm{a}^{54}$ & 2013 & RCT & Diabetic \\
\hline Cianci and Hunt ${ }^{56}$ & 1997 & CS & Diabetic \\
\hline Faglia et $\mathrm{al}^{57}$ & 1998 & CS & Diabetic \\
\hline Zgonis et $\mathrm{al}^{58}$ & 2005 & CS & Diabetic \\
\hline Fife et $\mathrm{al}^{59}$ & 2007 & CS & Diabetic \\
\hline Oubre et $a^{160}$ & 2007 & CS & Diabetic \\
\hline Ong $g^{61}$ & 2008 & CS & Diabetic \\
\hline Lyon $^{67}$ & 2008 & CS & Diabetic \\
\hline Bishop and Mudge ${ }^{62}$ & 2012 & CS & Diabetic \\
\hline Margolis et $\mathrm{a}^{63}$ & 2013 & CS & Diabetic \\
\hline Oliveira et $a^{66}$ & 2014 & CS & Diabetic \\
\hline Ueno et $\mathrm{al}^{47}$ & 2014 & CS & $\begin{array}{l}\text { Diabetic and } \\
\text { venous }\end{array}$ \\
\hline
\end{tabular}

Abbreviations: CT, controlled trial; ESWT, extracorporeal shock-wave therapy; RCT, randomized controlled trial; RT, randomized trial; CS, case series; HBO, hyperbaric oxygen.

nutritional intake, hydration, and optimal pressure area care and pressure relief remain the gold standard treatment for this category of wounds.

\section{Venous ulcers}

A single prospective trial on HBOT for venous ulcers randomized 16 participants to receive either hyperbaric oxygen $(\mathrm{n}=8)$ or sham treatment $(\mathrm{n}=8) .{ }^{44}$ The study was double-blind and the ulcers had been present for more than 1 year. The median patient age was 67 (range 42-75) years. Smokers and patients with other chronic conditions, such as diabetes, were excluded. The study participants were treated for 90 minutes at 2.5 ATA with either oxygen or air on 5 days per week for 30 treatments, and continued with compression bandaging throughout. Following 30 treatments, the mean reduction in wound area was $35.7 \%$ for patients in the HBOT group and $2.7 \%$ for those in the control group $(P<0.001)$.

Ueno et al published a retrospective study of 29 patients treated with HBOT in addition to standard wound care. ${ }^{47}$ The records of 14 males and 15 females with a mean age of $64.1 \pm 14.4$ years were reviewed. Ten patients had venous stasis ulcers and one had livedoid vasculopathy, a hyalinizing vascular disease resulting in thrombosis and ulceration of the legs. The remaining 18 patients had other chronic wounds, including 13 with diabetic ulcers. Subjects were treated at 2.8 ATA for 60 minutes with $100 \%$ oxygen once daily for 3-5 days a week. All patients received five or more HBOT sessions. The mean number of HBOT sessions was 22 (maximum 57). All but one of the venous stasis ulcers were on the leg. Six patients achieved healing or $>90 \%$ epithelialization at the end of HBOT and eight achieved a $>30 \%$ reduction in wound size, with wound healing being confirmed 6 weeks after completion of HBOT. Four patients showed a $<30 \%$ reduction in size, worsening of the wound, or an unhealed wound at 6-week follow-up. All patients with a poor outcome had diabetes and had undergone hemodialysis.

\section{Prospective studies in diabetic ulcers}

In one study, reduced amputation rates and improved wound healing were observed in patients who received HBOT $(n=18)$ when compared with more established treatment alone $(\mathrm{n}=10 ; P=0.001) .{ }^{48}$ A similar study conducted by Oriani et al observed a reduction in amputation rates and improved outcome in patients treated with HBOT $(n=62)$ when compared with the control arm of the trial comprised of patients unsuitable for HBOT because of contraindications to HBOT $(n=18) .{ }^{49}$ Although the distribution of major diabetic complications was similar between the groups, caution must be applied when comparing outcomes due to study design. In both studies, treatment was administered at either 2.8 ATA (antibacterial support) or 2.5 ATA (reparative effect). These treatment protocols are compliant with Undersea and Hyperbaric Medical Society guidelines. ${ }^{50}$ The number of treatments administered by Baroni et a ${ }^{48}(34 \pm 21.8$ daily treatments) and Oriani et $\mathrm{al}^{49}$ (72 \pm 29 HBOT sessions) were also in excess of the number routinely administered for diabetic foot ulcers, where 30-40 treatments are reported as common. ${ }^{51}$

Doctor et al studied diabetic patients with chronic foot ulcers to evaluate effect of HBOT as an adjunct to wound healing. ${ }^{37}$ Thirty hospitalized patients were randomized to receive either HBOT or conventional treatment. The number of patients allocated to each group was not reported, but all HBOT patients received a total of four treatments each of 45 minutes in duration at 3 ATA over a 2-week period, which is an unconventional treatment regime. The length of hospital stay, although shorter for the HBOT group, was not significantly significant compared to conventional treatment. As with the studies by Oriani et a $\mathrm{l}^{49}$ and Baroni et $\mathrm{al},{ }^{48}$ they 
reported a significant reduction in amputation above the ankle joint compared with the control. Control of wound infection by HBOT was also suggested, specifically in reducing Pseudomonas aeruginosa and Escherichia coli wound culture infections following HBOT treatment.

A significant reduction of major amputations in patients with diabetic foot ulcers treated with HBOT $(n=35)$ compared with control $(n=33)$ was reported by Faglia et al. ${ }^{39}$ Unlike Doctor et al, ${ }^{37}$ Faglia et $\mathrm{al}^{39}$ assessed ulcer severity using Wagner grading, and grade $2-4$ ulcers were included. ${ }^{10}$ Study reliability was improved by blinding of the surgeon assessing the need for amputation. As in the study by Oriani et al, an initial phase of HBOT at 2.5 ATA for 90 minutes daily was administered followed by a second phase at 2.2-2.4 ATA for 90 minutes. ${ }^{49}$ Patients received treatment 5 days per week, with an average of $38 \pm 8$ treatments per patient. The initial phase of treatment at 2.5 ATA was targeted at enhancement of antibacterial effect and rapid restoration of tissue oxygen tension, whereas the second phase was to stimulate fibroblast activity. In this study, Wagner grade 4 ulcers were the most prevalent, leading the authors to conclude that adjunctive HBOT is effective in decreasing major amputations in patients with severe diabetic foot ulcers.

A smaller study compared the healing rate of diabetic foot ulcers in five patients who received HBOT. ${ }^{52}$ The control group (also containing five patients) was comprised of patients refusing HBOT. This selection method is often considered ethically more acceptable than others because patients have the choice of whether to receive an intervention. However, those who consented to receive HBOT may have been more motivated toward positive health behavior. Both groups received standardized care, and assessment was by an individual blinded to group allocation. Thirty treatments were administered once per day, 5 days per week at 2.0 ATA, and unlike previous studies, follow-up continued for 4-6 months. The HBOT group demonstrated a significantly greater reduction in wound surface area compared with the control group. At follow-up, $80 \%$ of patients in the HBOT group had healed, with the fifth patient undergoing surgical coverage, whereas ulcers remained present in $80 \%$ of the control patients.

The long-term effect of HBOT in 38 patients with chronic diabetic foot ulcers and local hypoxia were investigated by Kalani et al. ${ }^{53}$ Patients were considered for inclusion if ulcers remained unhealed despite offloading and optimization of metabolic control (ulcer duration $>2$ months and $\mathrm{TcpO}_{2}<40 \mathrm{mmHg}$ ). Local access guided allocation to the treatment or control group after randomization proved difficult. Twenty-one patients were treated with standard care and 17 received adjunctive HBOT. At 3-year follow-up, 13 patients with ulcers in the HBOT group had healed, two patients had undergone below-knee amputations, and two had died, while ten patients in the control group had healed, seven had undergone below-knee amputation, three had died, and one showed improved ulcer healing. The patients in the HBOT group had greater initial ulcer surface areas but were also younger and so potentially had better healing prospects. The authors concluded that there was an accelerated healing rate and reduced amputation requirement in patients treated with HBOT, supporting previous studies.

A double-blind trial by Abidia et al investigated the therapeutic effect of HBOT on diabetic foot ulcers in patients with peripheral arterial disease. ${ }^{36}$ Eight patients in each group were randomized to receive either HBOT or sham treatment. They received five therapies per week at 2.4 ATA for 6 weeks, with HBOT patients receiving $100 \%$ oxygen and the control patients breathing air at pressure. Both treatments lasted for 90 minutes. Five patients from the HBOT group and one from the control group achieved healing after 6 weeks. The median decrease in wound area at 6-month follow-up was similar in both groups, ie, 100\% for the HBOT group versus 95\% for the control group. Five patients remained healed at 1 year in the HBOT group, whereas no healed wounds were present in the control group, leading the authors to suggest that HBOT has a prolonged effect. They also utilized the Short Form-36 and the Hospital Anxiety and Depression Scale to evaluate changes in quality of life and found no significant improvements in quality of life in the HBOT group compared with the control group.

A study by Kessler et al determined the effect of HBOT on Wagner grade 1-3 diabetic foot ulcers which had been present with no improvement for at least 3 months. ${ }^{40}$ Twenty-eight patients were randomized to receive either adjunctive HBOT $(n=15)$ or standard treatment alone $(n=13)$. Patients in the treatment arm received HBOT twice daily on 5 days per week for 2 weeks. Oxygen was administered at 2.5 ATA for 90 minutes, and follow-up continued for 2 weeks. The HBOT group demonstrated a significantly greater reduction in ulcer size at 2-week follow-up $(41.8 \% \pm 25.5 \%)$ compared with the control group $(21.7 \% \pm 16.9 \%)$, but 2 weeks later the reduction in ulcer size was comparable between the groups (48.1\% $\pm 30.3 \%$ for the HBOT group; $41.7 \% \pm 27.3 \%$ for the control group). Healing was observed in two patients who received HBOT but in no patients in the control group. The authors concluded that HBOT doubled the mean healing rate of diabetic foot ulcers, but follow-up suggested that improvements may be short-term. Only two trials have been 
reported using twice-daily HBOT which is not compliant with Undersea and Hyperbaric Medical Society guidelines, recommending treatment on a once-daily basis unless severe infection is present. ${ }^{50}$

A study of the effect of HBOT on infected diabetic foot ulcers present for at least 4 weeks has also been performed. ${ }^{38}$ Patients received adjunctive HBOT at 2-3 ATA for 90 minutes or standard care as determined through randomization. Twice-daily treatments were alternated with once-daily treatments for 20-30 days. This again is an unusual treatment regime and no justification for its use was provided. The mean follow-up duration was $92 \pm 12$ weeks. There were 50 patients in each group, but the HBOT group included significantly higher proportions of males, smokers, and obese patients. Patients treated with HBOT experienced better overall results than the control group; 33 healed without surgical intervention compared with none in the control group. Only four HBOT patients underwent amputations compared with 41 in the standard treatment group.

In 2010, Löndahl et al published a double-blind, randomized controlled trial reporting that the number of healed ulcers was doubled in their HBOT group at 1-year follow-up compared with their placebo group. ${ }^{41}$ Ninety-four patients with diabetic foot ulcers present for at least 3 months were randomized to receive HBOT $(n=49)$ or sham treatment $(n=45)$. All patients were treated at 2.5 ATA and, as in the study by Abidia et al, were administered either $100 \%$ oxygen or air for 90 minutes on 5 consecutive days per week for a total of 8 weeks. ${ }^{36}$ The treatment period was extended for up to 2 weeks for those patients who had missed therapies. A total of 38 HBOT patients and 37 control patients completed more than 35 treatment sessions (maximum 40 treatments), with $61 \%$ of the HBOT group and $27 \%$ of the control group achieving complete healing $(P=0.009)$. The largest difference in healing rate was reported at 9-month follow-up, indicating continued benefit after completion of HBOT. The number needed to treat to avert non-healing of a chronic foot ulcer was 4.2 based on intent-to-treat analysis and 3.1 for per protocol analysis. In a separate publication, Löndahl et al reported improved quality of life at 1-year follow-up as assessed using the Short Form-36. ${ }^{41}$

Wang et al randomized patients to receive extracorporeal shock-wave therapy twice a week for a total of six treatments ( $\mathrm{n}=41 ; 46$ ulcers) or undergo 20 HBOT sessions at 2.5 ATA ( $n=45 ; 47$ ulcers). Their results demonstrated a significantly greater number of healed ulcers in patients receiving extracorporeal shock-wave therapy $(P=0.003)$, although 20 HBOT sessions would not be considered a complete course of therapy in commonly accepted practice. ${ }^{43}$

In a study by Ma et al, 36 patients with Wagner grade 3 diabetic foot ulcers were randomized to HBOT or standard care alone. ${ }^{54}$ Distribution between the groups was not reported. Participants had ulcers for at least 3 months, a $\mathrm{TcpO}_{2}>30 \mathrm{mmHg}$, and were hospitalized for the 2-week study period. HBOT was administered twice daily 5 days a week. At day 14, following 20 HBOT sessions, there was a $42.4 \% \pm 20 \%$ reduction in wound area compared with $18.1 \% \pm 6.5 \%$ in the control group at the same time point. These differences were not observed at 7 days. Only one other prospective study administered HBOT twice daily, which is not a recommended regime. ${ }^{40}$

\section{Retrospective studies in diabetic ulcers}

Retrospective reviews comprise of analysis of routinely collected patient data for the investigation of treatment outcomes. Such observations lack the rigor of prospective studies and are heavily reliant upon the quality of existing documentation, which can frequently be incomplete and inconsistent. ${ }^{55}$ However, such analyses enable greater numbers of patients representative of the normal treatment population to be investigated.

Cianci and Hunt studied long-term outcomes in 41 patients with chronic Wagner grade 3 or 4 diabetic foot ulcers treated with vascular surgery and adjunctive HBOT over a 7-year period (1983-1990). ${ }^{56}$ Initial salvage had been achieved in $85 \%$ of these patients during the study period. In 1991, the wounds of 27 patients remained intact and one patient underwent a major amputation. The remaining 14 were lost to follow-up. In 1993, 22 of those 27 patients remained healed, suggesting a good long-term outcome for treatment of diabetic foot ulcers with vascular surgery and adjunctive HBOT. The authors suggested such a treatment regime to be potentially cost-effective, but the study size and inclusion criteria prevent generalizations to the entire population with diabetic foot ulcers.

Faglia et $\mathrm{al}^{57}$ reviewed patient outcomes between 1990 and 1993 in order to compare amputation rates with the results observed in two published prospective studies. ${ }^{39,48} \mathrm{Of}$ 115 patients treated, 51 were administered HBOT using the regime equivalent to that in the prospective studies. Patients received a mean number of $32 \pm 11$ HBOT treatments, and only seven required major amputations compared with 20 amputations in those who did not receive HBOT $(P=0.012)$.

A study assessing the relationship between HBOT and patient outcome following partial foot amputation over a 
10 -year period observed that $70 \%$ of patients (35 patients with 40 wounds) experienced healing or required no further surgical procedures to heal the wound following HBOT. ${ }^{58}$ A successful outcome was achieved, with a mean number of 20 treatments and 44 days to reach that endpoint compared with a mean of 16 treatments and 216 days in the failed group. The authors stressed that this study was intended to provide a starting point on which future research could be based. ${ }^{58}$

Fife et al investigated outcomes for 971 patients with diabetic lower extremity ulcers who received HBOT. ${ }^{59}$ They reported that 717 (73.8\%) patients had improved healing with 34 HBOT treatments. Patients who demonstrated no improvement received an average of 24 therapies. The authors suggested that discontinuation of apparently ineffective treatment generally occurred at this point. Fife et al highlighted that the main objectives of partial healing and stimulation of granulation and epithelialization are not always taken into consideration when the treatment outcome of HBOT is evaluated, even though the treatment is addressing chronic wounds that have shown no improvement for a significant time, with amputation being a realistic alternative option. ${ }^{59} \mathrm{In}$ their study, only 79 of 136 patients (58\%) with renal failure improved compared with 638 of 835 (76\%) without renal failure. Due to the difference in outcome with and without renal failure, the authors removed renal failure patients from the statistical analyses to avoid confounding the results. Smoking also appeared to adversely affect the outcome. Patients who received HBOT treatment once daily had better outcomes than those who were treated twice daily, possibly due to patients with more compromised limbs being treated more intensively rather than the higher frequency of treatment being less effective.

Oubre et al reviewed records of patients treated with HBOT for lower extremity wounds over a 6-year period to consider the outcomes of patients affected by different health factors including diabetes and smoking. ${ }^{60}$ Diabetics $(\mathrm{n}=37)$ were not found to have a significant difference in wound area reduction to that seen in non-diabetics $(n=37)$ using daily HBOT at 2.4 ATA for 6 weeks. The study failed to describe the breakdown of which diabetic patients achieved which outcome. Eighty-five non-healing lower extremity ulcers from 73 patients were included in the analysis. The authors found that smoking but not diabetes was associated with a poor outcome $(P<0.0001)$ and patients who fared better had higher $\mathrm{TcpO}_{2}$ readings and were younger.

Ong observed that a favorable outcome of $\geq 80 \%$ granulating tissue or partial or complete epithelialization following a course of HBOT was achieved in $71 \%$ of patients when retrospectively assessing diabetic foot ulcers treated over a 10-month period. ${ }^{61} \mathrm{~A}$ mean number of 20 treatments at 2.5 ATA were delivered once daily for 5 days per week. Of the 45 wounds described as foot ulcers, two were below-knee amputation wounds and a small unspecified number of wounds were leg ulcers.

Bishop and Mudge scrutinized patient records to assess what factors influence the outcomes of diabetic foot ulcers treated with HBOT at one center during a 2-year period $(n=30) .{ }^{62}$ In total, $73.3 \%$ of patients achieved a successful outcome at the end of HBOT and $70 \%$ remained successful 3 months later, at which time one patient (3.3\%) had a healed wound. At 3 months, $13.3 \%$ of patients were lost to follow-up and one patient (3.3\%) had a major amputation. Steroid therapy, previous minor amputation, type 1 diabetes, previous HBOT, larvae therapy, and application of interactive dressings had a significant relationship with poor outcome at the end of HBOT $(P<0.05)$. Three months later, peripheral vascular disease and previous minor amputation all had a significant influence on outcome, with affected patients more likely to be in the failure group $(P<0.05)$. The results of this study cannot be generalized due to the small sample size.

A longitudinal study including 83 centers in the USA assessed outcomes for patients treated over a 5.5-year period..$^{63} \mathrm{~A}$ total of 6,259 patients met the inclusion criteria, making this the largest study on diabetic wounds and HBOT. A total of 5,042 patients were excluded as they had healed, had amputations, or did not have a reduction in wound size of at least $40 \%$. Of these patients, 793 had HBOT, most often at 2 ATA (88.5\%), 5 days per week, and had 15-48 treatments. The researchers attempted to balance baseline covariates including age, sex, and wound age and size, and used proportional hazard models to minimize selection bias. It appeared that patients receiving HBOT were more likely to have an amputation and were less likely to heal; however, it is difficult to verify these results due to poor reporting and lack of detail. A number of comments have been made criticizing the methodology, conclusions, and general reporting of this study. ${ }^{64,65}$

A review of 25 patients with 26 wounds who received HBOT for Wagner grade 2 or higher diabetic ulcers found that $82 \%$ of wounds were healed or almost healed after HBOT. ${ }^{66}$ Twelve of the wounds included were foot amputation stump ulcers. Of the eleven diabetic foot ulcers, $82 \%$ were Wagner grade 3 or 4 . HBOT was administered five times a week at 2.4 ATA, with patients receiving 14-60 (median 36) treatments. Two patients required transmetatarsal amputation during treatment, but healed by the end of HBOT and three 
(13\%) had above-ankle amputations. One patient requiring amputation had end-stage renal disease. Fife et al also observed a poorer outcome in patients with renal disease. ${ }^{59}$ Ueno et al reviewed the records of 29 patients with chronic wounds treated with HBOT at their center, which was discussed in relation to venous ulcers earlier. ${ }^{47}$ Thirteen of the patients included had diabetic wounds. Six patients had more than $90 \%$ wound healing (one was diabetic) and eight patients had a $>30 \%$ reduction in wound size (three were diabetic). Four patients with diabetic ulcers had a poor response and had renal failure.

The results of these studies on HBOT for diabetic ulcers support the suggestion that HBOT used adjunctively with standard wound care improves healing, particularly for foot ulcers, and possibly reduces the need for major amputation. The long-term outcomes in patients were infrequently studied, but at centers where this was done, it was suggested that wounds often demonstrate a continued benefit from HBOT. Most studies had methodological flaws and small sample sizes. Further research with larger numbers of participants would be beneficial, with a focus on investigating the effect of different clinical outcomes in the short term and long term, particularly renal failure, ulcer severity, and once-daily versus twice-daily treatments. Although more than 40 HBOT were administered in some patient groups, 20-40 treatments was the most common number administered at 2-2.8 ATA. The regime is somewhat related to the aims of therapy in most cases.

\section{Conclusion}

Availability of hyperbaric oxygen varies around the world, and is more accessible in some countries than others. It must be used in combination with standard care rather as a therapy alone. Multidisciplinary team input is essential, and appropriate dressings, debridement, offloading, antibiotics, optimization of metabolic control, and adequate nutrition should all be considered and implemented as required. The cost of chronic wounds was discussed earlier in this paper. Research has demonstrated some benefit of HBOT for chronic wounds that are not responding to conventional treatment alone, with the majority of research concentrated on diabetic foot ulcers. In some countries, HBOT for diabetic foot ulcers now forms a routine part of the care pathway for patients not progressing in a timely manner or who are at risk of poor healing following surgical intervention. HBOT has been described as a costly treatment. However, the increasing costs of clinician time and more complex dressings may result in it being a more cost-effective intervention for non-healing wounds.
More robust research is required for a full assessment of the economics for these patient groups.

\section{Acknowledgments}

PE would like to acknowledge funding for some of the research reported in the paper from the Northcott Devon Medical Foundation and for PhD studentships generously supported by the DDRC Healthcare and the University of Exeter Medical School. The authors would also like to thank Mrs Susan Westoby for her secretarial assistance in the preparation of this manuscript.

\section{Disclosure}

The authors report no conflicts of interest in this work.

\section{References}

1. Richmond NA, Maderal AD, Vivas AC. Evidence-based management of common chronic lower extremity ulcers. Dermatol Ther. 2013;26(3): $187-196$.

2. Ruttermann M, Maier-Hasselmann A, Nink-Grebe B, Burckhardt M. Local treatment of chronic wounds: in patients with peripheral vascular disease, chronic venous insufficiency, and diabetes. Dtsch Arztebl Int. 2013;110(3):25-31.

3. Sen CK, Gordillo GM, Roy S, et al. Human skin wounds: a major and snowballing threat to public health and the economy. Wound Repair Regen. 2009;17(6):763-771.

4. Jones KR, Fennie K, Lenihan A. Evidence-based management of chronic wounds. Adv Skin Wound Care. 2007;20(11):591-600.

5. Kaplanski G, Marin V, Fabrigoule M, et al. Thrombin-activated human endothelial cells support monocyte adhesion in vitro following expression of intercellular adhesion molecule-1 (ICAM-1; CD54) and vascular cell adhesion molecule-1 (VCAM-1; CD106). Blood. 1998;92(4): 1259-1267.

6. Shaw FL, Winyard PG, Smerdon GR, Bryson PJ, Moody AJ, Eggleton P. Hyperbaric oxygen treatment induces platelet aggregation and protein release, without altering expression of activation molecules. Clin Biochem. 2009;42(6):467-476.

7. Kendall AC, Whatmore JL, Winyard PG, Smerdon GR, Eggleton P. Hyperbaric oxygen treatment reduces neutrophil-endothelial adhesion in chronic wound conditions through S-nitrosation. Wound Repair Regen. 2013;21(6):860-868.

8. Restrepo RD, Hirst KR, Wittnebel L, Wettstein R. AARC clinical practice guideline: transcutaneous monitoring of carbon dioxide and oxygen: 2012. Respir Care. 2012;57(11):1955-1962.

9. Fife CE, Buyukcakir C, Otto GH, et al. The predictive value of transcutaneous oxygen tension measurement in diabetic lower extremity ulcers treated with hyperbaric oxygen therapy: a retrospective analysis of 1,144 patients. Wound Repair Regen. 2002;10(4):198-207.

10. Wagner FW Jr. The dysvascular foot: a system for diagnosis and treatment. Foot Ankle. 1981;2(2):64-122.

11. Thom SR, Milovanova TN, Yang M, et al. Vasculogenic stem cell mobilization and wound recruitment in diabetic patients: increased cell number and intracellular regulatory protein content associated with hyperbaric oxygen therapy. Wound Repair Regen. 2011;19(2):149-161.

12. Thom SR, Mendiguren I, Hardy K, et al. Inhibition of human neutrophil beta2-integrin-dependent adherence by hyperbaric O2. Am J Physiol. 1997;272(3 Pt 1):C770-C777.

13. Almzaiel AJ, Billington R, Smerdon G, Moody AJ. Effects of hyperbaric oxygen treatment on antimicrobial function and apoptosis of differentiated HL-60 (neutrophil-like) cells. Life Sci. 2013;93(2-3):125-131. 
14. Gurdol F, Cimsit M, Oner-Iyidogan Y, Kocak H, Sengun S, YalcinkayaDemirsoz S. Collagen synthesis, nitric oxide and asymmetric dimethylarginine in diabetic subjects undergoing hyperbaric oxygen therapy. Physiol Res. 2010;59(3):423-429.

15. Dedi D, Prager T, Jacob R, Chan A, Fife C. Visual acuity chagnes in paritents undergoing hyperbaric oxygen therapy. Undersea Hyperb Med. 1998;25 Suppl:34.

16. Anderson B Jr, Shelton DL. Axial length in hyperoxic myopia. In: Bove AA, Bachrach AJ, Greenbaum LJ, editors. In: Underwater and Hyperbaric Physiology IX. Proceedings of the Ninth Symposium of Underwater And Hyperbaric Physiology. Bethesda, MD, USA: Undersea And Hyperbaric Medical Society; 1987.

17. Anderson B Jr, Farmer JC Jr. Hyperoxic myopia. Trans Am Ophthalmol Soc. 1978;76:116-124.

18. Weaver LK, Howe S. Normobaric measurement of arterial oxygen tension in subjects exposed to hyperbaric oxygen. Chest. 1992;102(4): $1175-1181$

19. Weaver LK, Howe S. Arterial oxygen tension of patients with abnormal lungs treated with hyperbaric oxygen is greater than predicted. Chest. 1994;106(4):1134-1139.

20. Yildiz S, Uluutku H, Gunay A, Yildirim I, Yildirim S, Gurbuz AK. The effect of hyperbaric oxygen therapy on the adverse effects of octreotide on wound healing. Eur J Gastroenterol Hepatol. 2004;16(5): 475-478.

21. Vrabec JT, Clements KS, Mader JT. Short-term tympanostomy in conjunction with hyperbaric oxygen therapy. Laryngoscope. 1998; 108(8 Pt 1):1124-1128.

22. Yarbrough OD, Behnke AR. The treatment of compressed air illness using oxygen. Journal of Indian Hygiene and Toxicology. 1939;21: 213-218.

23. Churchill-Davidson I, Sanger C, Thomlinson RH. High-pressure oxygen and radiotherapy. Lancet. 1955;268(6874):1091-1095.

24. Boerema I, Huiskes JW, Kroll JA, Kroon B, Lokin E, Meyne NG. High atmospheric pressure as an aid to cardiac surgery. Arch Chir Neerl. 1956;8(3):193-211.

25. Brummelkamp WH, Hogendijk J, Boerema I. Treatment of anaerobic infections (clostridial myositis) by drenching the tissues with oxygen under high atmospheric pressure. Surgery. 1961;49:299-302.

26. Smith G, Ledingham IM, Sharp GR, Norman JN, Bates EH. Treatment of coal-gas poisoning with oxygen at 2 atmospheres pressure. Lancet. 1962;1(7234):816-819.

27. Denham JW, Hauer-Jensen M. The radiotherapeutic injury - a complex 'wound'. Radiother Oncol. 2002;63(2):129-145.

28. Falanga V. Wound healing and its impairment in the diabetic foot. Lancet. 2005;366(9498):1736-1743.

29. Hung JH, Wang JH, Chen CY, Chao KC, Yang MJ, Hung J. Hyperbaric oxygen therapy for cesarean section wound in diabetes mellitus gravida. J Chin Med Assoc. 2008;71(7):373-376.

30. Hunt TK, Pai MP. The effect of varying ambient oxygen tensions on wound metabolism and collagen synthesis. Surg Gynecol Obstet. 1972;135(4):561-567.

31. Knighton DR, Silver IA, Hunt TK. Regulation of wound-healing angiogenesis - effect of oxygen gradients and inspired oxygen concentration. Surgery. 1981;90(2):262-270.

32. Milovanova TN, Bhopale VM, Sorokina EM, et al. Hyperbaric oxygen stimulates vasculogenic stem cell growth and differentiation in vivo. J Appl Physiol (1985). 2009;106(2):711-728.

33. Thom SR, Bhopale VM, Velazquez OC, Goldstein LJ, Thom LH, Buerk DG. Stem cell mobilization by hyperbaric oxygen. Am J Physiol Heart Circ Physiol. 2006;290(4):H1378-H1386.

34. Al Hadi H, Smerdon GR, Fox SW. Hyperbaric oxygen therapy suppresses osteoclast formation and bone resorption. J Orthop Res. 2013;31(11):1839-1844.

35. Kranke P, Bennett MH, Martyn-St James M, Schnabel A, Debus SE. Hyperbaric oxygen therapy for chronic wounds. Cochrane Database Syst Rev. 2012;4:CD004123.
36. Abidia A, Laden G, Kuhan G, et al. The role of hyperbaric oxygen therapy in ischaemic diabetic lower extremity ulcers: a double-blind randomisedcontrolled trial. Eur J Vasc Endovasc Surg. 2003;25(6):513-518.

37. Doctor N, Pandya S, Supe A. Hyperbaric oxygen therapy in diabetic foot. J Postgrad Med. 1992;38(3):112-114.

38. Duzgun AP, Satir HZ, Ozozan O, Saylam B, Kulah B, Coskun F. Effect of hyperbaric oxygen therapy on healing of diabetic foot ulcers. $J$ Foot Ankle Surg. 2008;47(6):515-519.

39. Faglia E, Favales F, Aldeghi A, et al. Adjunctive systemic hyperbaric oxygen therapy in treatment of severe prevalently ischemic diabetic foot ulcer. A randomized study. Diabetes Care. 1996;19(12):1338-1343.

40. Kessler L, Bilbault P, Ortega F, et al. Hyperbaric oxygenation accelerates the healing rate of nonischemic chronic diabetic foot ulcers: a prospective randomized study. Diabetes Care. 2003;26(8):2378-2382.

41. Löndahl M, Katzman P, Nilsson A, Hammarlund C. Hyperbaric oxygen therapy facilitates healing of chronic foot ulcers in patients with diabetes. Diabetes Care. 2010;33(5):998-1003.

42. Lin TF. The vascular effects of hyperbaric oxygen therapy in treatment of early diabetic foot. Undersea Hyperb Med. 2001;28 Suppl:67.

43. Wang CJ, Wu RW, Yang YJ. Treatment of diabetic foot ulcers: a comparative study of extracorporeal shockwave therapy and hyperbaric oxygen therapy. Diabetes Res Clin Pract. 2011;92(2):187-193.

44. Hammarlund C, Sundberg T. Hyperbaric oxygen reduced size of chronic leg ulcers: a randomized double-blind study. Plast Reconstr Surg. 1994;93(4):829-833.

45. Rosenthal AM, Schurman A. Hyperbaric treatment of pressure sores. Arch Phys Med Rehabil. 1971;52(9):413-415.

46. Hunter S, Langemo DK, Anderson J, Hanson D, Thompson P. Hyperbaric oxygen therapy for chronic wounds. Adv Skin Wound Care. 2010;23(3):116-119.

47. Ueno T, Omi T, Uchida E, Yokota H, Kawana S. Evaluation of hyperbaric oxygen therapy for chronic wounds. J Nippon Med Sch. 2014;81(1):4-11.

48. Baroni G, Porro T, Faglia E, et al. Hyperbaric oxygen in diabetic gangrene treatment. Diabetes Care. 1987;10(1):81-86.

49. Oriani G, Meazza D, Favales F, Pizzi GL, Aldeghi A, Faglia E. Hyperbaric oxygen therapy in diabetic gangrene. J Hyperb Med. 1990; 5(3):171-175.

50. Weaver LK. Hyperbaric oxygen therapy for carbon monoxide poisoning. Undersea Hyperb Med. 2014;41(4):339-354.

51. McMillan G, Glover M. The clinical and economic potential of hyperbaric oxygen therapy in the treatment of diabetic ulceration and other conditions. Int J Low Extrem Wounds. 2007;6(3):130-138.

52. Zamboni WA, Wong HP, Stephenson LL, Pfeifer MA. Evaluation of hyperbaric oxygen for diabetic wounds: a prospective study. Undersea Hyperb Med. 1997;24(3):175-179.

53. Kalani M, Jorneskog G, Naderi N, Lind F, Brismar K. Hyperbaric oxygen (HBO) therapy in treatment of diabetic foot ulcers. Long-term follow-up. J Diabetes Complications. 2002;16(2):153-158.

54. Ma L, Li P, Shi Z, Hou T, Chen X, Du J. A prospective, randomized, controlled study of hyperbaric oxygen therapy: effects on healing and oxidative stress of ulcer tissue in patients with a diabetic foot ulcer. Ostomy Wound Manage. 2013;59(3):18-24.

55. Parahoo K. Nursing Research, Principles, Processes and Issues. 2nd ed. Basingstoke, UK: Palgrave MacMillan; 2006.

56. Cianci P, Hunt TK. Long-term results of aggressive management of diabetic foot ulcers suggest significant cost effectiveness. Wound Repair Regen. 1997;5(2):141-146.

57. Faglia E, Favales F, Aldeghi A, et al. Change in major amputation rate in a center dedicated to diabetic foot care during the 1980s: prognostic determinants for major amputation. J Diabetes Complications. 1998; 12(2):96-102.

58. Zgonis T, Garbalosa JC, Burns P, Vidt L, Lowery C. A retrospective study of patients with diabetes mellitus after partial foot amputation and hyperbaric oxygen treatment. J Foot Ankle Surg. 2005;44(4): 276-280. 
59. Fife CE, Buyukcakir C, Otto G, Sheffield P, Love T, Warriner R 3rd. Factors influencing the outcome of lower-extremity diabetic ulcers treated with hyperbaric oxygen therapy. Wound Repair Regen. 2007; 15(3):322-331.

60. Oubre CM, Roy A, Toner C, Kalns J. Retrospective study of factors affecting non-healing of wounds during hyperbaric oxygen therapy. $J$ Wound Care. 2007;16(6):245-250.

61. Ong M. Hyperbaric oxygen therapy in the management of diabetic lower limb wounds. Singapore Med J. 2008;49(2):105-109.

62. Bishop AJ, Mudge E. A retrospective study of diabetic foot ulcers treated with hyperbaric oxygen therapy. Int Wound J. 2012;9(6):665-676.

63. Margolis DJ, Gupta J, Hoffstad O, et al. Lack of effectiveness of hyperbaric oxygen therapy for the treatment of diabetic foot ulcer and the prevention of amputation: a cohort study. Diabetes Care. 2013;36(7): 1961-1966.
64. Carter MJ, Fife CE, Bennett M. Comment on: Margolis et al. Lack of effectiveness of hyperbaric oxygen therapy for the treatment of diabetic foot ulcer and the prevention of amputation: a cohort study. Diabetes Care. 2013;36(8):e131.

65. Hawkins GC. Comment on: Margolis et al. Lack of effectiveness of hyperbaric oxygen therapy for the treatment of diabetic foot ulcer and the prevention of amputation: a cohort study. Diabetes Care. 2013; 36(8): 129.

66. Oliveira N, Rosa P, Borges L, Dias E, Oliveira F, Cassio I. Treatment of diabetic foot complications with hyperbaric oxygen therapy: a retrospective experience. Foot Ankle Surg. 2014;20(2):140-143.

67. Lyon KC. The case for evidence in wound care: investigating advanced treatment modalities in healing chronic diabetic lower extremity wounds. J Wound Ostomy Continence Nurs. 2008;35(6):585-590.
Chronic Wound Care Management and Research

\section{Publish your work in this journal}

Chronic Wound Care Management and Research is an international, peer reviewed, open access, online journal publishing original research, reviews, editorials, and commentaries on the causes and management of chronic wounds and the major issues related to chronic wound management. Topics also include chronic wounds as comorbidities to other

\section{Dovepress}

conditions, patient adherence to therapy, and the economic burden of chronic wounds. The manuscript management system is completely online and includes a very quick and fair peer review system, which is all easy to use. Visit http://www.dovepress.com/testimonials.php to read real quotes from published authors.

Submit your manuscript here: http://www.dovepress.com/chronic-wound-care-management-and-research-journal 